\title{
Knud Thomsen
}

Paul Scherrer Institut, Switzerland

\section{THE OUROBOROS MODEL EMBRACES ITS SENSORY-MOTORIC FOUNDATIONS AND LEARNS TO TALK}

\begin{abstract}
The Ouroboros Model proposes a brain inspired cognitive architecture including detailed suggestions for the main processing steps in an overall conceptualization of cognition as embodied and embedded computing. All memories are structured into schemata, which are firmly grounded in the body of an actor. A cyclic and iterative data-acquisition and -processing loop forms the backbone of all cognitive activity. Ever more sophisticated schemata are built up incrementally from the wide combination of neural activity, concurrent at the point in time when the memory is established; i.e., distinct representations are accrued. Later on, an entire schema can be reinstated from diverse subsets of its constituent features. In order to decode or compile ever more elaborate constructions, extant building blocks are concatenated. They are serially linked via common or "connection-attributes" of different representations and symbols, and they are employed for serial perception, processing and action, in particular, also for language production. At various levels, commonalities between different schemata lead to a similar preferred use of their respective tokens and subsequently shared categorizations. Rules, for the concatenation of words, akin to the ones governing chemical reactions, can be abstracted. As special kinds of symbols, words and word-classes along with the whole grammar of a language can thus be seen as a direct consequence of the processes outlined in the Ouroboros Model. Strong emphasis is put on the dimension of time over short intervals during active experience and performance, and, in particular, during interactions with other agents.
\end{abstract}

Keywords: schemata, serial iterative processing, anticipations, consistency, communication

\section{Introduction}

The Ouroboros Model is a biologically inspired cognitive architecture which is built around an algorithmic backbone of iterative and self-referential processing (Thomsen, 2010a). A key tenet sees all memory content as organized into meaningful chunks, called schemata. Many of them are laid down as a kind of snapshot of all activations in a living brain at particular points 
in time. Following a second route, concepts can also be distilled from statistical regularities and co-occurrences of arbitrary feature representations. In addition, effective schemata can be assembled on the fly from combinations of preexisting building blocks like (parts of) already available schemata as a response to an (external) trigger or prescription (Thomsen, 2010b). Especially in the first case, all principally possible content, e.g., sensory percepts, bodily reactions, action dispositions, and associated preexisting conceptualizations are tied together as they are jointly activated at the same time; thus also the most abstract schemata and any derived/distilled abstract symbols are originally grounded in the body of the agent.

According to the Ouroboros Model, embodiment is indispensable for the working of (situated, extended, embedded as well as abstract) cognition; the body serves not only as a substrate or vehicle for action but as a fundamental constituent of the cognitive functioning of any individual and autonomous human or animal actor in the real world. All activity, and, in particular, all signs and tokens are connected to their roots in the real external material world through the senses (the body) of the actor; they are grounded (Harnad, 1990). This becomes especially visible with metaphors (Citron \& Goldberg, 2014).

It has been described elsewhere how, according to the Ouroboros Model, embodiment and self-reflective abstraction built upon it lay the foundations for human consciousness (Thomsen, 2011a).

\section{The Time-Course of Action}

On the Ouroboros Model, all activity progresses along a principle sequence, implementing a basic processing loop:

- ... anticipation,

- action/perception,

- evaluation,

- anticipation, ...

These steps or stages are concatenated into a full repeating cycle, and any current activity builds on the hitherto available content, i.e., on the previously achieved results, which are incorporated while the activity progresses, all in a sense akin to the old alchemists' tail-devouring serpent called the Ouroboros. Evaluation is the core process, "consumption analysis" checks how well open slots in the selected schema can be filled with incoming data, i.e., it monitors the consistency between expectations and actual subsequent 
activations; all action is biased and directed by that intermediate outcome, in particular, by any discrepancies detected (Thomsen, 2011b).

One important proposition of the Ouroboros Model is that all activations stored at a particular point in time are later also effective together. When exciting one feature, an entire schema will be activated and reinstated. Pattern completion, thus implemented, forms part of the very basis of our heritage and survival: it certainly was better for our ancestors who glimpsed the tail of a tiger in the high grass to assume that an entire animal was there and try to flee rather than waiting for sensible confirmation and the tiger's teeth.

It can be claimed that already Otto Selz $(1913,1922)$ has outlined similar principles. He coined the term schema, and he was probably among the first to note that a complete schema can be activated already by a subset of its constituents with anticipations pointing to some missing components. Schemata can be stacked and nested, i.e., structures at one level can function as components for a next higher-level entity. Anticipations are at the core of an orderly problem solving process. As Otto Selz observed, errors occur in cases when only part of the relevant information is effectively used. He also described the effects of sentence-schemata, which fit perfectly with what will be worked out in the following; e.g., in a simple version: "who did what to whom" (Arunachalam \& Waxman, 2014). Open slots in schemata, principally, bias input, which promises to fit.

\section{Conceptual Basis}

This short paper is not meant to present a full-blown theory of language but rather an attempt is made to take a step back and grasp an overall, albeit coarse-grained, picture. It is a sketch of how the proposed algorithmic backbone and the underlying structures and processes could match with simple observations and selected extant theories concerning features of languages and their use. (Communicative) action unfolds on the foundations of existing (memory-) structures, and new such compositions are laid down as its consequence. This iterative process began at a truly fundamental level with the first living organisms and, over the course of evolution, reached the highest currently known heights in sophisticated scientific reasoning and the arts.

Depending on the point of view and on what features in the resulting web of concepts are treated as essential, very different components, preferred starting points, or facets can be declared as fundamental. Examples 
range from abstract ideas as independently existing Platonic entities to action dispositions and sensory-motoric contingencies as exemplified by Kevin O'Regan (2013). The Ouroboros Model holds that often the effectively resulting conceptualizations are rather equivalent, merely highlighting different aspects and attributes of complex structured compound schemata, all grounded and actually made possible by the bodily existence of an agent. Qualia, like Plato's ideas or mathematical symbols, are but examples of specific abstractions, which lay emphasis on particular selected features of a schema. Grounding is especially essential for qualia, and it constitutes the basis and commonly contributes significant parts to the content of any effective schema in an overall functional account of cognition. By definition, this means privileged private access for the owner for experiencing and communicating.

A crucial point is that the basic principles of memory organization as well as processing stay the same, independent of the types of the linked constituting attributes, which can also stem from very different spheres. Any neural activation can be incorporated into new entities and contexts; the resulting approximately self-similar hierarchy of concepts is not a strict one, and still, rather well-distinct building blocks are accrued.

In particular, self-monitoring does not require any completely different ingredients for meta-cognition: the very same processing steps working with just another set of elaborate concepts added, i.e., ones referring selfreflectively to the actor, do the trick (Thomsen, 2011a). The claimed benefits of a meta-cognitive loop, "MCL", for the robustness of intelligent systems can safely be taken as staying the same (Haidarian et al., 2010). A repeated sequence of "Note-Assess-Guide" is a practical shortcut describing what the Ouroboros Model does all the time; powerful general-purpose anomalyhandling strategies are nothing but particular schemata, easily learnable (as abstractions) from experience or instruction.

Active visual observation and directed search can serve as a simple example for the unfolding of action; exploration of the environment and its relevant features can always be conceptualized as mutual interaction between an actor and her surroundings or context. A case in point are affordances of objects, which direct attention and even provoke specific (re)actions of an agent (O'Regan, 2013).

Notwithstanding the importance of interaction with the surrounding inanimate environment, other agents in a community are recognized as providing truly fundamental input and indispensable scaffolding for any cognitive behavior. 


\section{Representations}

Memories, according to the Ouroboros Model, are laid down in the form of structured schemata. They allow meaningful action at subsequent occasions. Linking actual sensory data with the prevailing context, relating in particular also to the actor, they share a common general format for any representation of whatever content.

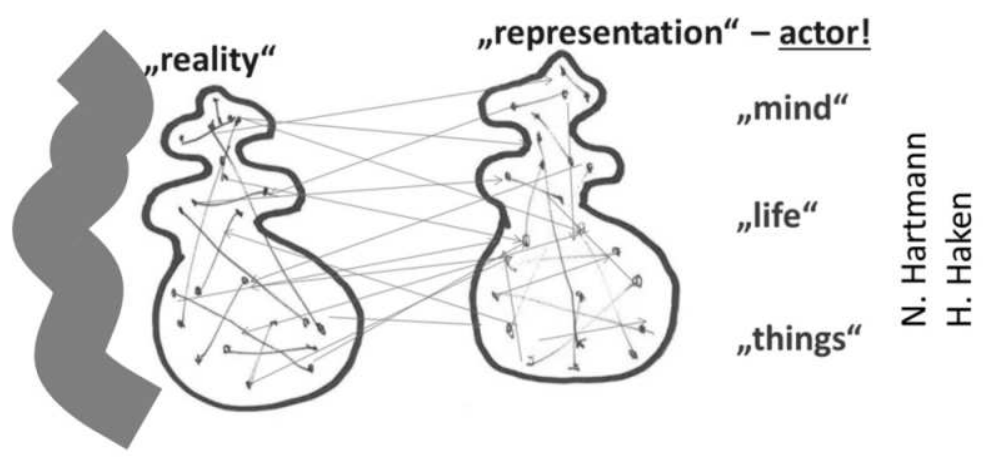

Figure 1. Overview sketch of the correspondence of the grounding of any "real thing" with internal structure (left) and partly matching links in the a conceptual multi-modal representation (middle); any schematic representation is, first of all, a data structure in and for an individual actor. Following Nicolai Hartmann, different realms/layers can be distinguished, which can be directly mapped to separated layers as devised by the synergetics of Hermann Haken. The grey area on the far left simply is out of (current) reach; in a sense, it does not even exist for the actor.

The Ouroboros Model describes how useful schemata are distilled from experience as a consequence and under the control of the flow of activity constrained by the relevant boundary conditions at any stage (Thomsen, 2010b). (Almost) arbitrarily selected constituents and links can be taken as fundamental, many classifications are possible; the one of Nicolai Hartman (1935-1950) into different layers (of abstraction) appears to be especially suitable, and it matches nicely with the insights of synergetics as devised by Hermann Haken (1977). The sketch in Figure 1 depicts that some correspondence between an outside reality and an individual actor must hold - otherwise the actor would not have come along so far. The Ouroboros Model can be seen as a continuation and extension of the successful approach taken by evolution (e.g., with the interplay: hoof $\leftrightarrow$ steppe), with an added component of directionality due to the inherent focusing of attention and memory resources to where the need arises and where the content is potentially most valuable and helpful in the future (Thomsen, 2011b). 
In the extreme, schemata even determine what exists - for an individual agent or a whole group sharing (objective as well as cultural) constraints and convictions; for the case of declarations by a law-giving authority, this can be true literally (Searle, 2014). "Newspeak" and, pointing in the same direction, the emphasis on using politically correct expressions are attempts at actively forming a correct world by allowing only "desirable" representations (Orwell, 1949).

A special class of representations includes auditory features, where sounds are associated with a concept. These auditory features can stand for the whole implicated schema, which also comprises dedicated motor programs for producing exactly these sounds. Commonly, such tokens are called (spoken) words. Signals, signs, and letters are similar entities with the emphasis more on the visual domain.

At a fundamental level, any bodily action has to respect certain limitations, like that one cannot lift and lower one's arm at the same time (Cotterill, 2000). The Ouroboros Model takes this as an example of a truly universal constraint, which principally demands some form of consistency checking and ensuing inhibition of contradictory actions and activations. Avoiding contradictions and inconsistencies lies at the heart of the behavior of any efficient and considerate (cognitive) agent. This applies to bodily action and in direct extrapolation/continuation also to mental activities. Difficult movements as well as careful deliberations are regularly performed in steps, one after the other.

The rich information offered by natural scenes and complex objects is likewise absorbed serially. Canonical scan paths over faces followed by viewers have been observed for decades (Noton \& Stark, 1971). According to the Ouroboros Model, they can be understood as a consequence of one prominent feature like an eye triggering the search for a next, usually closely related, feature, such as a mouth or an ear. Moving on to conceptual levels higher up in the hierarchy of schemata, the influence of catalogue descriptions on the visual scanning and the perception of works of art has been demonstrated recently (Bałaj, 2013).

Given the general capacity limits of working memory and, in particular, the parcellation of all memory entries into coherent structures, i.e., schemata, in order to construct larger assemblies and to communicate them, one has to combine existing chunks, and at a certain level of complexity, this is only possible in a primarily serial fashion. As long as main constituents and their relations are preserved, e.g., available in some canonical arrangement of the features, a representation can be easily encoded and decoded. Time is essential for an effective succession with the 
focus on parts, constituents and facets, one after the other. In any case, the processes are complex, and a singular or unitary strictly linear account, where, e.g., a completely clear and well-set meaning is just translated into words, certainly is too simple (Pickering \& Garrod, 2013). Thoughts are also developed while speaking, with activity on different levels progressing in parallel. It has been shown that the content of an utterance can be manipulated without the speaker noticing it afterward, even approving a changed meaning as his own (Lind et al., 2014).

\section{Collaborative Communication}

Language here is first and foremost taken as a means to make a mental edifice of one actor, i.e., a meaningful construction built up from schemata, available to another. Here, a static world is presupposed; in reality, this is generally not the case, and the outside world will undergo changes over time, in particular, also such caused by (embodied) agents.

It appears mandatory that for any dialog a minimum reference to some common ground, "reality", is shared by both parties (Thomsen, 2013; Jacquette, 2014). There can be neither agreement nor disagreement in the absence of some minimally sufficient referential intentionality.

Not all levels of schemata are equally important for language; the features depicted in the "upper" levels of Figure 1 appear to be most relevant for the present investigation. With abstraction and through the transfer from one agent to another, there unavoidably occur shifts of emphasis and losses of direct connections to the material world.

Schemata and concepts (and, in the end, words representing them) come in families; they share many (important) features inside a category and some less across category boundaries (Wittgenstein, 1953). The first observation means that, at a coarse level, one concept can be used to take over the function and place of another, related one. The second point leaves a way of naturally linking distinct schemata mediated by common or easily interlocking features; see Figure 2.

Languages concatenate signs and tokens at diverse levels, which exhibit much resemblance. Basic language universals and linguistic biases are obviously ground-laid at birth; certain (combinations of) phonemes are much more common than others in any given language, or even in all known languages, and certain words and word-classes are preferentially occurring together (in a specific order) (Gómez et al., 2014; Cinque, 1999); see cartoon in Figure 3. 


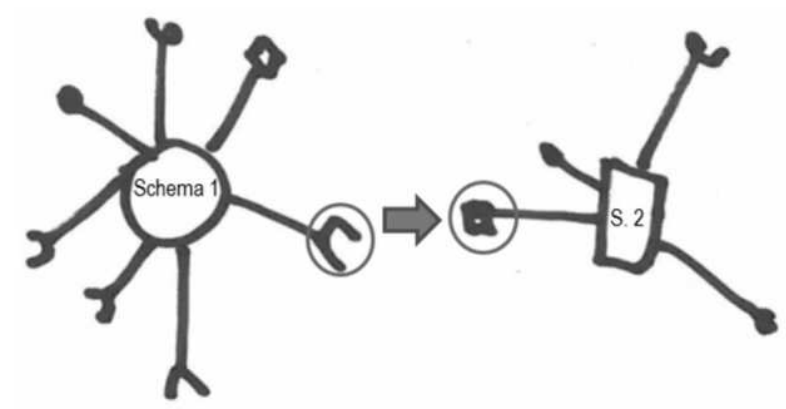

Figure 2. Cartoon of two schemata with fitting connection/docking features

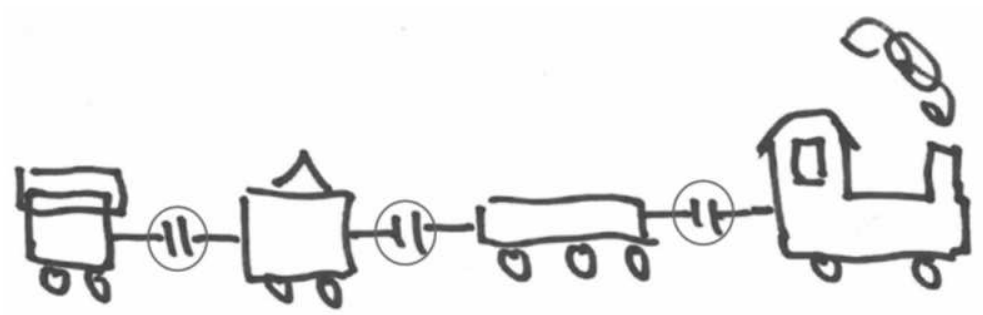

Figure 3. A compound schema (sentence) as built up from different constituents with engaging features

It is claimed that the same basic processes are decisive, irrespective of the particular content; priming is effective at the semantic level as well as relating to all features involved in preferred combinations, i.e., abstractions from repeated co-occurrences and common use. Structural/syntactic priming is explained as a manifestation of the general phenomenon in the field of languages' (surface) structures (Ledoux et al. 2007; Reitter et al., 2011).

What follows can be taken as an extension as well as a specification of verb valency in syntactic theory and can coarsely be summarized as a "chemical" model of syntax and grammar:

At the level of words and, similarly, at levels underneath and above, units comprise features usable for coupling, "connection sites". Preferably, recruiting these specific points, they can be concatenated to build up larger constructs like molecules are synthesized from single atoms.

In straight-forward extension of serial processing at the component level, schemata, for which there is a sign (e.g., a word), are linked in a series involving specific docking sites, which means the employment of peculiar slots for connections. They are implemented like any other feature, which, for 
example, codes semantic content or the associated phonemes; see Figure 2. As particular combinations occur repeatedly and in different contexts but with similar meaning, such connections can take shape as abstracted units of their own, i.e. norms for pronunciation or abstracted grammatical rules. This fits neatly with the semantic feature hypothesis, which postulates that word meanings are acquired componentially (Clark, 1973).

Children generally learn verbs later than concrete nouns as they denote relational features and meaning as compared to direct combinations of features for more specific real-world entities (Genter, 1978). Verbs often are abstractions, and they are applicable wider than simple concrete nouns.

The same processes are hypothesized to lead to the invention of function words when an original meta-expression is directly expressed on the word level; it is postulated that basically the same steps can lead to different constructions, but with similar function, in quite diverse languages.

Regularities in a language, i.e., grammar and syntax, arise from shared characteristics of the available units for encoding content (based in the end on relations in the relevant world), and the associated processes for assembling and concatenating these symbols during acts of communication.

On the one hand, as a result of repeated use, regularities crystallize over time into abstractions; on the other, they provide scaffolding and set the frame for the successful encoding of new information, applicable in an analog way on many different levels of language. As any schema, also grammatical ones excite expectations; they provoke anticipations on many diverse levels (Selz, 1913, 1922).

Linear encoding (and decoding) of semantic content is possible only when the sounds are articulated clearly enough and when meaningful words are strung together according to accepted rules, i.e., not deviating too much from common practice. One needs to obey the rules, i.e., comply with the constraints of a language to some extent in order to express oneself freely. All discourse relies on some shared content; it cannot work without a minimum of common reference, the (portion/view of the) world jointly deemed relevant; see Figure 4.

Rules, according to the Ouroboros Model, are abstractions describing dynamic equilibria. Languages live; they are subject to many influences and fashions. At any time, they are characterized by exceptions and constant reshaping with distinctions being not so clear-cut; words and grammatical features are newly coined and they also disappear, e.g., as in the current case of the genitive in German.

Relating the "chemical" view sketched above to established theories and approaches in any detail goes beyond what is possible in this short 


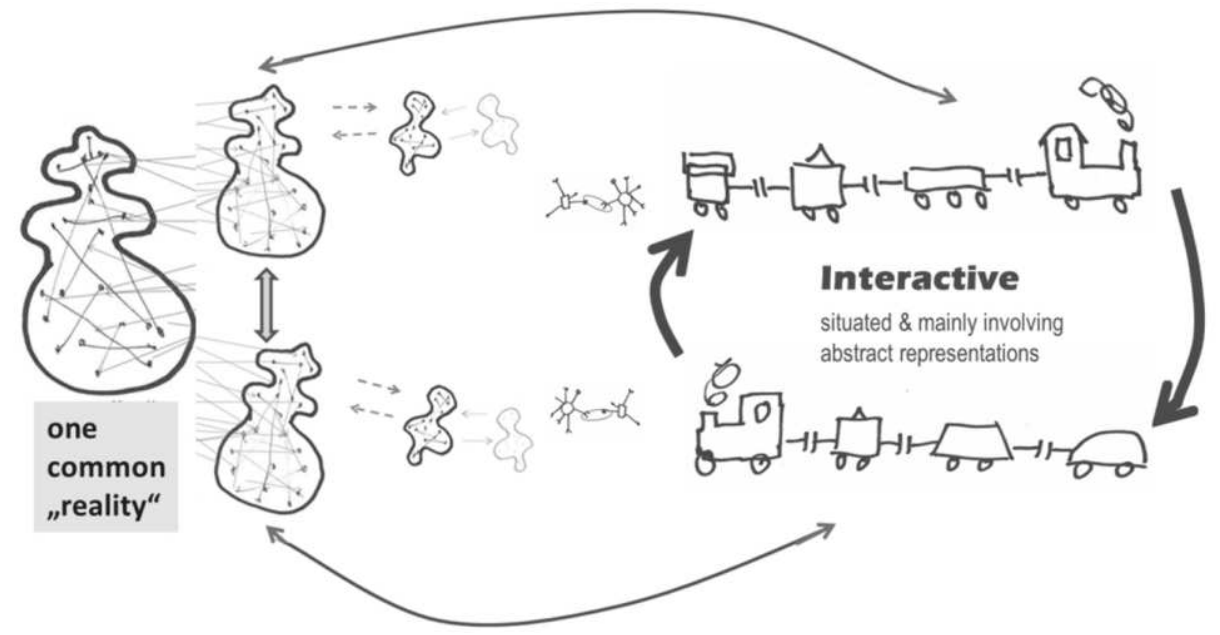

Figure 4. Overview sketch of the grounding of any conversation and the linear coding of meaning in discourse in front of a static world, see text for details

paper. To mention just one issue in addition to the hinted similarity with Ludwig Wittgenstein's ideas (1953), a seemingly fitting link can be made to the "focal" approach recently advocated by Andrej Kibrik (2012). The conception outlined here addresses both discrete, continuous, and also "nonlinear" holistic facets of language.

Figure 4 depicts a schematic overview of actual communication, not really restricted to the talking of two persons to each other; it works identically in non-verbal contexts. As noted before, only a static outside world is considered here.

In any case, successful dialog seems to rely on a number of components and the iterative and emergent coordination of mutual references and exchanges. Being embedded in common reality, or at least making reference to one, the two partners each have their individual representation thereof; these might be slightly different but cannot be completely disjunct. Each person has a repertoire of concepts/schemata and tokens/words, which stand for the relevant facets of the respective reality. To formulate a sentence, the speaker strings together words to cover and convey the intended information and meaning, a message. Details are determined by common use and features related to the coupling of the employed schemata on diverse levels; conventions are effective. The responder, guided by the same habits and standards, decodes the utterance by linking the tokens to the best fitting schemata available to her-which is easier with more shared reality (-representations). 
Differences concerning the (independently by the disputants and especially mutually) perceived applicability and relevance of dimensions and the emphasis on diverging features and attributes trivially explain how it is possible that people talk at cross-purposes and cannot understand each other.

Even in unchanging outside reference settings, varying loops occur in many forms, inside the agents and in the interplay between them. An immediate reply might just focus on one word or even a sound, whereas a profound answer to an elaborate statement would only be possible after taking into account wide-spread content and manifold relations, following all the steps as sketched before. Iteratively, the partners build up some increased common comprehension, each experiencing an enhancement and augmentation of their perceptions, opinions, and understanding compared to before.

The production of language and its comprehension according to the Ouroboros Model are tightly interwoven in an interplay between input and output relying on the same memory resources and basic processing steps (Thomsen, 2013). Some resemblance of the conceptualization as sketched in figure 4 can be spotted with the integrated theory of language as recently proposed by Martin Pickering and Simon Garrod, who similarly stress the importance of predictions (2013). Prediction here is another word for anticipation, the term used by Otto Selz; schemata are activated, and slots are opened and then (hopefully) filled, both for the conceptualizations of each partner in the dialog and also in their communicative exchange unfolding over time, all with considerable overlap and working on many diverse levels in parallel during oral conversation. The many involved strata extend all the way between single phonemes and the (possibly concealed) intentions of a speaker.

In a dialog, the important exchange and key interplay is between the disputants; their behavior is mutually coordinated. Taking turns, they deliver input, i.e., some type of affordances as highlighted by Joanna RączaszekLeonardi (2013), for the partner, and they decipher what has been said (indicated, meant). The dialog partners alternately provide their counterpart with serially unfolding constructions, kindling anticipations of perceptions and signals (words) to come.

Witness to the importance of embodiment, the external loop via a partner in a conversation as shown in Figure 4 can also be short-circuited, i.e., restricted to (the head of) one actor in soliloquy, with still some of the effects present. Talking to oneself and thinking slowly, explicitly, probably pronouncing words loudly, activates compounded schemata additionally 
and can thus help to better come to grips with a difficult situation - not only for small children. Gesturing or directly involving the hands serves the same purpose and has been shown to greatly benefit abstract problem solving (Rączaszek-Leonardi, 2013; Guthrie, 2013). Co-speech gestures as embodied manual action quite generally are tightly coupled to language and have a marked facilitative impact on language production and understanding (Lucero et al., 2014; Willems \& Hagoort, 2007).

Essential to the Ouroboros Model, anticipations are always only defined in relation to actually available data. It has been sketched how the Ouroboros model can be conceptualized as an implementation of Bayesian processing (Thomsen, 2011b); in this view, priors are contributed by the previously established concepts, i.e., schemata, and hypotheses are generated in this context when integrating newly arriving data.

There is ample evidence for Bayesian cue integration drawing on a plethora of possibly relevant sensory channels in healthy people, which also offers ways of explaining deviant outcomes under the special conditions of mental diseases (Lind et al., 2014; Moore \& Fletcher, 2012). As to the ownership of utterances in particular, related deficits observed with schizophrenia, "thought disorder", can be interpreted as showing a blurred distinction between syntactic and semantic dimensions, plagued by general problems with a disorganized memory, sense of ownership, and disturbed cue integration (Kupferberg \& Caplan, 2003).

It has been claimed that in recent linguistic theories the distinction between lexical items and traditional rules of grammar is vanishing with unification operations taking center stage and occurring in parallel at the semantic, syntactic, and phonological levels of processing (Hagoort, 2005). Pragmatic dimensions certainly come in addition. As an alternative, or maybe rather refinement of unification, a maintenance/prediction hypothesis has recently been presented (Lewis et al., 2015). For example, gamma power increases in EEGs are observed as specifically linked to the actual occurrence of a highly predictable lexical item, i.e., the filling-in of an empty slot in a compound schema.

Fully in accord with the tenets of the Ouroboros Model, novel verbs can be learned best when distinct features, syntactic frame, and semantic content conspire in synergy in complete sentences (Arunachalam \& Waxman, 2014).

In any serious dialog, participants can rightly demand from each other a certain minimum of consistency (Mackenzie, 2014). Rules can be broken in many different and subtle ways. The Ouroboros Model features at its core the constant monitoring of consistency as the central engine for advance- 
ment, meaning amongst others, and not only in communicative situations, checking the adherence to rules.

Discrepancies, all deviations from an anticipation, direct attention to the implied feature(s) of a schema and eventually lead to enhanced personal and, finally, conscious involvement (Thomsen, 2010a, 2011a, 2011b). Recent results obtained with neuroimaging can be naturally interpreted as confirming this, and they allow linking that general statement to ways of elaborate formulating. William Shakespeare made extensive use of functional shifts, i.e., utilizing nouns as verbs; a semantically appropriate word is employed in a syntactically inappropriate role. Occurrences of this stylistic device have been found to trigger surprise and enhanced mental processing in wider-than-normal spread brain regions, in fact forcing a listener into a more active role for integrating the meaning of what has been said (Keidel et al., 2013).

Interestingly, this particular effect would not work in Chinese where an object and the associated action can be denoted by the same word.

Other vehicles for pointed expression would be (to varying extent, with subtle differences between cultures): irony, sarcasm, analogy or wit, to name just a few, which are all claimed to enhance processing by first making direct and literal understanding (a little) more difficult.

Metaphors aim at easing understanding by starting from a familiar domain; still, their decoding, which requires some type of transfer, would benefit from a similar logic (Schneider et al., 2014). Typically, metaphorical sentences are literally false; according to the Ouroboros Model, this excites attention. Metaphorical expressions often allude to bodily experience; e.g., taste-related words engage corresponding cortical areas and also emotional centers more than literal words in otherwise identical sentences (Citron \& Goldberg, 2014).

People are born story-tellers and story-understanders, i.e., we strive to assign bits of information into a consistent mental model (schema). Understanding a text requires coherence-building between successive sentences. The presence of a lexical connection (cohesion) aids in establishing coherence (Ferstl \& von Cramon, 2000). Simple incoherent sentence pairs have been shown to elicit smaller activation than coherent sentence pairs in the left frontomedian wall, posterior cingulate, and precuneal regions. Without cohesion, the detection of incoherence was found to be more difficult. An area in the left inferior prefrontal cortex was responding sensitively to the difficulty of the task with higher activation in more demanding conditions. It appears that right hemisphere resources are only recruited additionally when the dominant left hemisphere is lesioned or unusual or erroneous stim- 
uli are presented; this fits nicely with the tenets of the Ouroboros Model, which holds that activation spreads to recruit more resources when no easy fit is found quickly.

A marked ending of a sentence brings an idea, a schema, to a close. In order to further maintain and process the associated activation, additional effort is required; there is inhibition of return to be overcome and also the inhibition of alternatives, which prevails once one interpretation has been selected (Thomsen, 2010a). Depending on the material affected, i.e., the level of abstraction concerned, details are different and, in particular, time scales (Tydgat et al., 2012). Phonetic features certainly should be relatively easy to access for repeated use. The interplay between facilitation and inhibition is guiding the unfolding of a conversation and of cognition in general at diverse levels. Concurrent parallel activities are nested and coordinated but not in simple synchrony; short time scales apply to lower levels and long ones to higher abstractions. Without being clearly marked as "consumed", lingering representations interfere with the production of subsequent respective units.

\section{Competitive Communication}

Emphasizing coherence and consistency, one has to stay attentive to the fact that friendly collaboration and mutual benefit is not always the primary aim of communicative acts.

Looking at a wider frame of somehow interdependent (mental) processes changing with respect to and in response to each other, the Ouroboros Model certainly offers not the only theory, which comprises some type of processing loops.

For example, the "OODA loop", which distinguishes the stations Observe-Orient-Decide-Act, can be seen as a related theory, albeit swapping the general aim of cooperation and mutual understanding for competition, even fighting (Boyd, 1976). Results are in full accord with the Ouroboros Model, which in terms of consumption analysis does offer an algorithmically much clearer account than merely referring to "feedback" (Thomsen, 2010a).

The cycles of constant quality-control and improvement, "PDCA cycles", which consist of the stages Plan-Do-Check-Act, fit into the same mold; here, feedback is delivered by customers and the market (Shewhart, 1939). There is considerable practical and commercial interest in 
such models; a web search for "PDCA" yields many different shades and promotions by diverse companies (Moen \& Norman, 2009). Repeated refinement and continuous improvement ("kaizen") match perfectly with the iterations of the Ouroboros Model, especially in periods or settings without major resets. The results achieved in the frameworks of the OODA-loop and the PDCA-loop for competitive settings nicely complement and contrast the considerations relating to a general collaborative spirit.

In short, these conceptualizations with iterative cyclic activity at their very core can be seen as special cases of the Ouroboros Model.

Successful dynamic action in all communicative contexts relies on shared grounding and (conceptual) building blocks as well as on taking everything relevant, in particular, the actions of opponents/partners, fully into account. A difference in sign results from whether the goal of the involved agents is the establishing or the disruption of cycles of mutual anticipation, overall agreement, understanding, and consistency. As quite often in human affairs, the real picture is not black and white, but there are shades of grey in between, maybe even different colors. Communication often is not either only collaborative or competitive or straight controversial. There are many nuances, with multifaceted dimensions added, like in, amongst others, propaganda, deception, and pretense, which all involve the deliberate distortion of "plain" schemata.

An option also exists to act in a communicative setting that can be called a "third way out". Examples can be drawn from "non-answers" of experienced politicians. Seen from the perspective of the Ouroboros Model in this case, a response to a question is formulated but not really addressing the raised points, denying to fill-in the open slots. Also here, there is a continuum: under certain circumstances one rightfully first has to explain what the perceived most relevant and important issues are; and, there are situations where making statements only loosely related to the real topic and sidetracking the attention, usually by adding more aspects, dimensions, and conditions, is a means to avoid giving a direct and somehow unpleasant answer. In the framework of a pragma-dialectical perspective Corina Andone (2014) recently analyzed a paradigmatic example of confrontation and political accountability with very much the same results.

Quite general and simple: an interrogator usually is only really content with the answer to a question when the missing information is indeed provided and meeting the expectations, i.e., consumption analysis yields: no contradictions or inconsistencies (detected), and no more unfilled slots at the demanded level of relevance and detail. 


\section{Discussion}

What is the added value of the Ouroboros Model? In one word: crossdisciplinary integration.

Based on a minimum of core ingredients, i.e., a memory structured in chunks or packets, schemata, and a self-referential monitoring process, "consumption analysis", the Ouroboros Model offers an overarching framework extending from the bodily level of embedded agents to their highest mental abstractions and their use in communication. A possible way is sketched to (let), systematically and self-controlled, evolve an autonomous cognitive system, which reasons while relying on basic principles of constraint satisfaction and adhering to an overall Bayesian approach (Thomsen, 2011b). In real-time, priorities are assigned and decisions are taken on the currently best available basis. Predictions take a central stage; they make the wheel turn, by directing attention towards inconsistencies and gaps. Resources are efficiently allocated to where they are needed most. This (as good as possible) allows the bridging over of previous barriers and disputes, showing a way forward. Communication, and, in particular, human language, offers most versatile and open-ended tooling for exactly this, while its basic structures and building blocks, it is suggested, themselves evolved from this very process.

The Ouroboros Model in fact can be understood as being based on enactivism, and the described structures and mechanisms also entail it: first, active movement and subsequently cognition arise through the dynamic interaction between an active organism and its environment. The (facets of the) environment, which are relevant to an actor, determine the representational as well as action-capabilities of such agents, who in turn shape and to some extent freshly create that frame of reference. Organisms do not passively receive information; perception is an active process. Schemata derived from previous experience are fundamental; they enable predictions and planned action. In an effective hierarchy of schemata a rather tight coupling to an outside reality exists close to the bodily grounding level; higher up the ladder of abstraction, the freedom for (still not arbitrarily) co-constructed knowledge and conventions expands for human individuals allowing definitions via socio-linguistic interactions. For any meaningful discourse, a minimum set of communalities between individual partners is indispensable at the level deemed as relevant.

All of this is explained with a most parsimonious approach in a selfconsistent and self-reflectively consistent manner. The ground-laid structures and processes auto-catalytically foster their permanence and their 
suitable extension. Important to note, observing the distinctions between the involved entities and, in particular, their specific timing, no vicious cycle arises.

It has been claimed that reflecting back on the roots in neural implementation, the Ouroboros Model can shed light on the use of the majority of neurons in a human brain, i.e., in the cerebellum (Thomsen, 2014), while along the same lines, intricate issues concerning intelligence and consciousness can be tackled (Thomsen, 2011a).

\section{Conclusion and Outlook}

Starting from some simple observations like the organization of memory content into distinct coherent structures, schemata, and the fundamental functional requirement for embodied agents to monitor their actions for coherence and consistency, the Ouroboros Model sketches a self-reflective picture of successful communication. In cooperative settings, as well as during competition or fight, a number of shared components are important for the iterative and emergent coordination of mutual references and exchanges. Embedded in one common reality, the involved partners each have their individual, but inevitably in a sense similar, representation thereof.

The Ouroboros Model offers a natural way of explaining how very rich multi-modal representations are compiled and tightly interweaved and how, starting from different venture points and diverse triggers, similar structures, courses of action, and outcomes can result.

Regularities in a language are claimed to arise from shared characteristics of the available units for encoding content, e.g., shared connection points inherent to schemata and distilled from common practice. As one consequence, the process of the concatenation of symbols during speech appears to follow grammatical rules, which in turn provoke anticipations and aid the decoding by the recipient just the same as and in parallel to semantic priming.

It goes without saying that in the here presented sketch a great many aspects and functions of language have been left out for later work. Just to mention a few topics, it will have to be investigated whether questions can be so simply understood as just adding a well-circumscribed open slot to an otherwise completely defined schema, similar in that sense to orders or warnings. A trivial prediction would be that learning is facilitated at the fringe of content already mastered. It is claimed that the Ouroboros Model can explain pretense, lies, and jokes, and it can shed light on social func- 


\section{Knud Thomsen}

tions as well as the respective roles of cultures and ideologies, in particular, with respect to communication and the exchange of ideas. As a hypothesis related to an implementational level, a stutter might be caused by faulty inhibition of return, an ingredient which is deemed essential to orderly cognitive processing and communication according to the Ouroboros Model.

Human languages are multi-faceted, recursive, and inherently openended and ambiguous. Understanding grammatical rules as abstractions hints a way to further extending the frame. The rules of formal logic are congruously seen by the Ouroboros Model as abstractions, too; only in a restricted "clean", well-defined setting can there be a clear distinction between whether a predication is true or false (when keeping to finite local realms).

On a modest scale, applicable for this work, the question of relevance (with the presupposed answer: "yes") is, whether the sketched proposals could be of any use for attaining some more-encompassing understanding and, to that aim, for directing further, more detailed, studies.

\section{Acknowledgments}

This work has benefited substantially from inspiring presentations and discussions at the first Avant conference in Torun and the subsequent exchange of emails and ideas with several colleagues. I would also like to thank an unknown referee for very insightful and helpful comments on an earlier version of this manuscript.

\section{R E F E R E N C E S}

Andone, C. (2014). Maneuvering with the burden of proof: confrontational strategies in dealing with political accountability. Studies in Logic, Grammar and Rhetoric, 36, 59-78.

Arunachalam, S., \& Waxman, S. R. (2014). Let's see a boy and a balloon: Argument labels and syntactic frame in verb learning. Learning and Development, in press.

Bałaj, B. (2013). Visual scanning of figurative and abstract painting. Research on naïve in the field of art. Paper presented at Trends in Interdisciplinary Studies, 1st International Avant Conference, Torun, Poland.

Boyd, J. R. (1976). Destruction and creation. U.S. Army Command and General Staff College. Retrieved from http://www.goalsys.com/books/documents/ DESTRUCTION_AND_CREATION.pdf

Cinque, G. (1999). Adverbs and functional heads. Oxford: Oxford University Press.

Clark, E. V. (1973). What's in a word? On the child's acquisition of semantics in his first language. In T. E. Moore (Ed.), Cognitive development and the acquisition of language (pp. 65-110). New York: Academic Press. 
Cotterill, R. (2001). Cooperation of the basal ganglia, cerebellum, sensory cerebrum and hippocampus: possible implications for cognition, consciousness, intelligence and creativity. Progress in Neurobiology, 64, 1-33.

Citron, F. M., \& Goldberg, A. E. (2014). Metaphorical sentences are more emotionally engaging than their literal counterparts. Journal of Cognitive Neuroscience, in press.

Ferstl, E. C., \& von Cramon, D. Y. (2001). The role of coherence and cohesion in text comprehension: an event-related fMRI study. Cognitive Brain Research, $11,325-340$.

Genter, D. (1978). On relational meaning: The acquisition of verb meaning, Child Development, 49, 988-998.

Gómez, D. M., Berent, I., Benavides-Varela, S., Bion, R. A. H., Catarossi, L., Nespor, M., \& Mehler, J. (2014). Language universals at birth. Proc. Nat Acad. Sc., 111, 5837-5841.

Guthrie, L. (2013). The hands that do the thinking: Interactivity in mental arithmetic. Paper presented at Trends in Interdisciplinary Studies, 1st International Avant Conference, Toruń, Poland.

Hagoort, P. (2005). On Broca, brain and binding: a new framework. Trends in Cognitive Sciences, 9, 416-423.

Haidarian H. S, Dinalankara, W., Fults, S., Wilson, S., Perlis, D., Schmill, M., Oates, T., Josyula, D. S., \& Anderson, M. L. (2010). The metacognitive loop: An architecture for building robust intelligent systems. AAAI Fall Symposium: Commonsense Knowledge, Vol. FS-10-02 of AAAI Technical Report. AAAI.

Haken, H. (1977). Synergetics: an introduction; nonequilibrium phase transitions and self-organization in physics, chemistry and biology. Berlin: Springer.

Hartmann, N. (1935-1950). Ontologie. 4 Bände. Berlin: Walter de Gruyter.

Harnad, S. (1990). The symbol grounding problem. Physica D, 42, 335-346.

Jacquette, D. (2014). Collective referential intentionality in the semantics of dialogue. Studies in Logic, Grammar and Rhetoric, 36, 143-159.

Keidel, J. L., Davis, P. M., Gonzalez-Diaz, V. Martin, C. D., \& Thierry, G. (2013). How Shekespeare tempests the brain: Neuroimaging insights. Cortex, 49 , 913-919.

Kibrik, A. A. (2012, June). Non-discrete effects in language, or the critique of pure reason 2. Paper presented at The Fifth International Conference in Cognitive Science, Kaliningrad.

Kupferberg, G. R., \& Caplan, D (2003). Language dysfunctioon in schizophrenia. In R. B. Schiffer, S. M. Rao, \& B. S. Fogel (Eds.): Neuropsychiatry, 2nd ed. (pp. 444-466). Philadelphia, PA: Lippincott Williams and Wilkins.

Ledoux, K., Traxler, M. J., \& Swaab, T. Y. (2007). Syntactic priming in comprehension: Evidence from event-related potentials. Psychological Science, 18, $135-143$. 
Lewis, A. G., Wang, L., \& Bastiaansen, M. (2015). Fast oscillatory dynamics during language comprehension: Unification versus maintenance and prediction? Brain $\mathcal{E}_{3}$ Language, in press. doi:10.1016/j.bandl.2015.01.003

Lind, A., Hall, L., Breidegard, B., Balkenius, C., \& Johansson, P. (2014). Speaker's acceptance of real-time speech exchange indicates that we use auditory feedback to specify the meaning of what we say. Psychological Science, 25(6), 1198-1205. doi: 10.1177/0956797614529797

Lucero, C., Zaharchuk, H., \& Casasanto, D. (2014). Beat gestures facilitate speech production. In P. Bello, M. Guarini, M. McShane, \& B. Scassellati (Eds.), Proceedings of the 36th Annual Conference of the Cognitive Science Society (pp. 898-903). Austin, TX: Cognitive Science Society.

Mackenzie, J. (2014). From speech acts to semantics. Studies in Logic, Grammar and Rhetoric, 36, 121-142.

Moen, R., \& Norman, C. (2009). Evolution of the PDCA cycle. Retrieved on 4 April 2014 from http://kaizensite.com/learninglean/wp-content/uploads/2012/09/ Evolution-of-PDCA.pdf

Moore, J. W., \& Fletcher, P. C. (2012). Sense of agency in health and disease: A review of cue integration approaches. Consciousness and Cognition, 21, $59-68$.

Noton, D., \& Stark, L. (1971). Eye movements and visual perception. Scientific American, 224, 35-43.

O'Regan, J. K. (2013). Why things feel the way they do: The sensorimotor approach to understanding phenomenal consciousness. Trends in Interdisciplinary Studies, 1st International Avant-Conference, Torun, Poland.

Orwell, G. (1949). Nineteen eighty-four. London: Secker \& Warburg.

Pickering, M. J., \& Garrod, S. (2013). An integrated theory of language production and comprehension. Behavioral and Brain Sciences, 36, 1-64.

Rączaszek-Leonardi, J. (2013). Thinking with Hands, Eyes, Things and Others. Trends in Interdisciplinary Studies, 1st International Avant Conference, Torun, Poland.

Reitter, D., Keller, F., \& Moore, J. D. (2011). A computational cognitive model of syntactic priming. Cognitive Science, 35, 587-637.

Schneider, S., Rapp, M. A., Haeußinger, F. B., Ernst, L. H., Hamm, F. Fallgatter, A. J., \& Ehlis, A.-C. (2014). Beyond the N400: Complementary access to early neural correlates of novel metaphor comprehension using combined electrophysiological and haemodynamic measurements. Cortex, 53, 45-59.

Searle, J. H. (2014). The Structure and functions of language. Studies in Logic, Grammar and Rhetoric, 36, 27-40.

Selz, O. (1913). Über die Gesetze des geordneten Denkverlaufs, erster Teil. Eine experimentelle Untersuchung. Stuttgart: Speemann.

Selz, O. (1922). Über die Gesetze des geordneten Denkverlaufs, zweiter Teil. Zur Psychologie des produktiven Denkens und des Irrtums. Bonn: Cohen. 
Shewhart, W. A. (1939). Statistical method from the viewpoint of quality control. Washington, DC, The Graduate School, the Department of Agriculture, pp. 155.

Thomsen, K. (2010a). The Ouroboros Model in the light of venerable criteria. Neurocomputing, 74, 121-128.

Thomsen, K. (2010b). Concept formation in the Ouroboros Model. Third Conference on Artificial General Intelligence, AGI 2010, Lugano, Switzerland.

Thomsen, K. (2011a). Consciousness for the Ouroboros Model. Journal for Machine Consciousness, 3, 163-175.

Thomsen, K. (2011b). The Ouroboros Model, selected facets. In C. Hernández et al. (Eds.), From brains to systems (pp. 239-250). New York: Springer.

Thomsen, K. (2013). The Ouroboros Model embraces its sensory-motoric foundations. Paper presented at Trends in Interdisciplinary Studies, 1st International Avant Conference, Torun, Poland.

Thomsen, K. (2014). The cerebellum according to the Ouroboros Model, the "interpolator hypothesis". Journal of Communication and Computer, 11, 239-254.

Tydgat, I., Diepedaele, K., Hartsuiker, R. J., \& Pickering, M. J. (2012). How lingering representations of abandoned context words affect speech production. Acta Psychologica, 140, 218-239.

Willems, R. M., \& Hagoort, P. (2007). Neural evidence for the interplay between language, gesture and action: A review. Brain 83 Language, 101, 278-289.

Wittgenstein, L. (1953). Philosophische Untersuchungen. Kritisch-genetische Edition (J. Schulte, Hrsg.). Frankfurt: Wissenschaftliche Buchgesellschaft. 